\section{(- OPEN ACCESS}

\title{
Cortical thinning is associated with disease stages and dementia in Parkinson's disease
}

\author{
Mojtaba Zarei, ${ }^{1,2}$ Naroa Ibarretxe-Bilbao, ${ }^{3,4,5}$ Yaroslau Compta, ${ }^{4,6}$ Morgan Hough, ${ }^{7}$ \\ Carme Junque, ${ }^{3,4}$ Nuria Bargallo, ${ }^{8}$ Eduardo Tolosa, ${ }^{4,6}$ Maria Jose Martí ${ }^{6}$
}

\begin{abstract}
- Additional material is published online only. To view please visit the journal online (http://dx.doi.org/10.1136/ jnnp-2012-304126).

For numbered affiliations see end of article.

\section{Correspondence to}

Dr Maria Jose Martí,

Parkinson's Disease and Movement Disorders Unit, Neurology Service, Institut Clínic de Neurociències (ICN), Institute of Biomedical Research August Pi i Sunyer (IDIBAPS), Hospital Clínic de Barcelona, c.Nillarroel 170, Barcelona, Catalonia 08036 Spain; mjmarti@clinic.ub.es
\end{abstract}

Received 13 September 2012 Revised 21 January 2013 Accepted 11 February 2013 Published Online First 5 March 2013

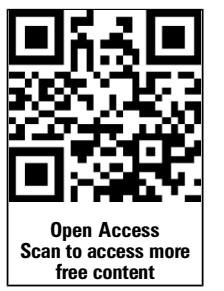

To cite: Zarei $\mathrm{M}$, IbarretxeBilbao N, Compta Y, et al. J Neurol Neurosurg Psychiatry 2013;84:875-882.

\section{ABSTRACT}

Objective To investigate the pattern of cortical thinning in Parkinson's disease (PD) across different disease stages and to elucidate to what extent cortical thinning is related to cognitive impairment.

Design Ninety-six subjects including 39 controls and 57 PD patients participated in this study. PD subjects were divided into three groups (early, $n=24$; moderate, $n=18$; with dementia, $n=15$ ). High field structural brain MRI images were acquired in a 3T scanner and analyses of cortical thickness and surface were carried out. Vertex-wise group comparisons were performed and cortical thickness was correlated with motor and cognitive measures.

Results We found a positive correlation between MiniMental State Examination scores and cortical thickness in the anterior temporal, dorsolateral prefrontal, posterior cingulate, temporal fusiform and occipitotemporal cortex. Unified Parkinson's Disease Rating Scale-III (motor subsection) scores showed a robust negative correlation with caudate volumes. We found that disease stage in PD was associated with thinning of the medial frontal (premotor and supplementary motor cortex), posterior cingulate, precuneus, lateral occipital, temporal and dorsolateral prefrontal cortex. Discriminant analysis and a receiver operating characteristics approach showed that mean cortical thickness and hippocampus volume have $80 \%$ accuracy in identifying PD patients with dementia. PD stage and PD dementia can be characterised by a specific pattern of cortical thinning.

Conclusions We conclude that measuring cortical thickness can be useful in assessing disease stage and cognitive impairment in patients with PD. In addition, cortical thickness may be useful in identifying dementia in PD.

\section{INTRODUCTION}

Parkinson's disease (PD) is a progressive neurodegenerative disorder that presents with motor symptoms but may lead to cognitive impairment and dementia. ${ }^{12}$ This progression from motor to cognitive dysfunction is likely related to the ascending spread of $\alpha$-synuclein deposition (Lewy bodies and neurites) from lower brainstem nuclei to cortical areas, following the stages proposed by Braak and Del Tredici. ${ }^{3}$ Braak stages have been associated with cognitive status and disease stage, but not with disease duration, age at disease onset, or age at death. ${ }^{4}$ Furthermore, cortical involvement by other pathologies, as Alzheimer's type pathology, has also been suggested as a potential contributor to PD-related dementia (PDD). ${ }^{5-7}$ Thus, some type of cortical damage is likely to be critical in cognitive dysfunction in PD.

Neuroimaging has provided powerful methods to assess neurodegenerative diseases in vivo. High resolution MRI can accurately measure changes in cerebral structures and function. Previous MRI studies using voxel-based morphometry (VBM) showed a correlation of grey matter (GM) atrophy with disease progression and cognitive impairment in PD. ${ }^{8}{ }^{9}$ More recent studies have shown that cortical thinning occurs in $\mathrm{PD}^{10}$ and that it is associated with disease duration. ${ }^{11}$ Cortical thinning in PD without dementia has been reported to be related to cortical folding abnormalities and GM volume reductions. ${ }^{12}$ These three different measures (cortical thickness, cortical folding and GM volume) provide complementary and related information on neurodegenerative changes in PD. However, surface-based measures of cortical folding, and especially cortical thickness, are seemingly more sensitive than VBM for identifying regional cortical thinning associated with PD. ${ }^{12}$

Recent studies support involvement of the hippocampus as a biomarker of cognitive decline and progression to dementia in PD. ${ }^{13}{ }^{14}$ However, it remains unclear how cortical thinning is related to cognitive impairment and disease stage in PD. The identification of a specific pattern of cortical thinning associated with disease stage and the evolution of cognitive impairment would add to the available evidence concerning cortical involvement in PD and constitute a potential biomarker of dementia in PD.

Corticometry has been successfully used for the study of other diseases and ageing. ${ }^{15-17}$ Corticometry has three main advantages over VBM: (i) corticometry uses geometry to carry out inter-subject registration which results in much better matching of homologous cortical regions than volumetric techniques; (ii) corticometry allows us to look separately at the two components of cortical volume (thickness and surface area); and (iii) the target that corticometry uses for registration (the white matter surface geometry) is completely invariant to GM atrophy, so GM changes would not result in a different registration.

In this study, we used MRI corticometry on a relatively large cohort of PD patients at different disease stages (from newly diagnosed PD to PDD cases). We compared PD subjects at different disease stages to examine whether: (i) the pattern of cortical thinning extends with advancing disease stage; (ii) the motor state in PD correlates with subcortical GM loss or cortical thinning; (iii) there is a 
linear relationship between cognitive impairment and cortical thinning; and (iv) cortical thinning is a useful quantitative measure to classify patients as having dementia.

\section{METHODS}

\section{Participants}

Subjects were recruited from the Parkinson's Disease and Movement Disorders Unit (Hospital Clínic Barcelona) as part of a research programme on the clinical, neuropsychological, neurochemical and imaging correlates of PD, with a number of reports having been already published from this dataset. ${ }^{12}$ 18-20 This study was approved by the local institutional ethics committee.

A total of 96 subjects were studied: 24 early PD (EPD) patients (Hoehn \& Yahr (H\&Y) stage $\leq 2$, disease duration $\leq 5$ years, absence of motor fluctuations and absence of dementia), 27 moderate PD patients ( $\mathrm{H} \& \mathrm{Y}$ stage $2-4$, disease duration $>5$ years and absence of dementia), 20 PD patients with dementia (H\&Y stage $>3$, disease duration $>5$ years and presence of dementia) and 39 healthy controls. All provided informed written consent to participate in this research programme. All participants in the study were right handed. Control subjects $(n=39)$ had no neurological signs on examination, scored $>26$ on the Mini-Mental State Examination (MMSE), and were recruited from friends and spouses of the patients and matched for age, gender and level of education with the PD subjects. A neurologist (MJM or YC) made the diagnosis of definite PD based on the UK Parkinson's Disease Society Brain Bank diagnostic criteria for PD. ${ }^{21}$ To date, no indication of atypical parkinsonism has been detected in any of our PD patients according to the clinical diagnostic criteria for parkinsonian disorders appraised by the Movement Disorders Society Task Force. $^{22}$ H\&Y staging of the PD patients was distributed as follows: stage $1, n=4$; stage $1.5, n=6$; stage $2, n=18$; stage 2.5 , $\mathrm{n}=1$; stage $3, \mathrm{n}=14$; stage $4, \mathrm{n}=11$; and stage $5, \mathrm{n}=3$. Dementia was defined according to the Diagnostic and Statistical Manual of Mental Disorders, Fourth Edition, Text Revision (DSM-IV-TR) and the Movement Disorder Society (MDS) criteria for PDD (19) with special attention being paid to the 1-year rule to distinguish PDD from dementia with Lewy bodies. In the EPD group, three patients were taking no medication and 21 were on the following anti-Parkinsonian treatment at the time of investigation: MAO-B inhibitor $(n=7)$, L-dopa monotherapy $(n=5)$, dopamine agonist monotherapy $(n=3)$ or a combination of L-dopa and dopamine agonist $(n=6)$. None of the EPD patients were receiving psychoactive medication at the time of the study. In the moderate PD group and the group with PD and dementia, several of the patients were taking antidepressants, antipsychotics and cholinesterase inhibitors. All patients were clinically stable and no patient was asked to change her/his medication for this study.

\section{Clinical assessment}

The severity of motor symptoms was assessed by means of the motor section of the Unified Parkinson's Disease Rating Scale (UPDRS-III), and by H\&Y classification of disease stage. The MMSE test was used to assess global cognitive status and the Beck Depression Inventory (BDI-II) to assess depression. Evaluation of visual hallucinations $(\mathrm{VH})$ was carried out using the Spanish version of the Neuropsychiatric Inventory (NPI) (hallucinations subscale) and a structured interview designed at our hospital covering the type (visual, auditory, tactile and olfactory) and temporal aspects of the hallucinations (time of the day, frequency and duration). The $\mathrm{VH}$ in our PD sample consisted of well-formed images of people, faces or animals. Hallucinations occurred only in the visual modality while patients were alert and had their eyes open and did not present a secondary auditory component. The clinical and demographic data of the participants are shown in table 1 .

\section{Image acquisition}

Images were acquired using a TIM TRIO 3T scanner (Siemens, Germany). A set of high-resolution three-dimensional T1-weighted images was acquired with a MPRAGE sequence $(\mathrm{TR} / \mathrm{TE}=2300 / 2.98 \mathrm{~ms} ; \mathrm{TI}=900 \mathrm{~ms} ; 256 \times 256$ matrix, $1 \mathrm{~mm}$ isotropic voxel).

\section{Image analysis}

Image analysis was carried out at the FMRIB Centre, Oxford, using Freesurfer software. The technical details of cortical reconstruction and volumetric segmentation procedures are described in prior publications. ${ }^{23} 24$ Briefly, this processing includes motion correction, removal of non-brain tissue using a hybrid watershed/surface deformation procedure, automated

Table 1 Clinical and demographic characteristics

\begin{tabular}{|c|c|c|c|c|c|}
\hline & YHC & $\mathrm{OHC}$ & EPD & MPD & PDD \\
\hline $\mathrm{n}$ & 24 & 15 & 24 & 18 & 15 \\
\hline Gender (M/F) & $17 / 7$ & $6 / 9$ & $16 / 8$ & $12 / 6$ & $5 / 10$ \\
\hline Age (years) & $57.6 \pm 8.9$ & $71.8 \pm 8.0$ & $56.5 \pm 9.0$ & $69.6 \pm 6.3$ & $73.2 \pm 7.6$ \\
\hline Side onset $L / R / B$ & 0 & 0 & $10 / 12 / 2$ & $7 / 9 / 2$ & $5 / 6 / 4$ \\
\hline UPDRS-III & 0 & 0 & $15.0 \pm 3.7$ & $32.9 \pm 16.9$ & $35.5 \pm 8.8$ \\
\hline$H \& Y$ & 0 & 0 & $1.8 \pm 0.6$ & $2.9 \pm 0.8^{*}$ & $3.9 \pm 0.8^{*}$ \\
\hline LEDD & 0 & 0 & $327 \pm 365$ & $1016 \pm 409$ & $735 \pm 290$ \\
\hline Disease duration (years) & 0 & 0 & $3.2 \pm 1.9$ & $10.7 \pm 4.9$ & $11.0 \pm 7.7$ \\
\hline MMSE & $29.8 \pm 0.4$ & $29.1 \pm 1.1$ & $29.3 \pm 1.8$ & $27.7 \pm 1.8$ & $18.3 \pm 3.8^{* *}$ \\
\hline VH & 0 & 0 & 0 & 3 & 8 \\
\hline BDI-II & $4.5 \pm 5.1$ & $6.2 \pm 6.9$ & $6.75 \pm 4.8$ & $8.9 \pm 6.1$ & $9.3 \pm 4.6$ \\
\hline
\end{tabular}

*Indicates significant difference between MPD and PDD ( $F=12, p<0.001)$.

**Indicates significant difference between MPD/PDD and $\mathrm{OHC}(\mathrm{p}<0.0001)$.

Values are mean \pm SD.

B, both; BDI-II, Beck Depression Inventory-II; EPD, early Parkinson's disease; H\&Y, Hoehn and Yahr staging; L, left; LEDD, levodopa equivalent daily dose; MMSE, Mini-Mental State Examination; MPD, moderate Parkinson's disease; OHC, old healthy control; PDD, Parkinson's disease with dementia; R, right; UPDRS-III, Unified Parkinson's Disease Rating Scale (motor section); VH, visual hallucination; YHC, young healthy control. 
Talairach transformation, segmentation of the subcortical white matter and deep GM volumetric structures, intensity normalisation, tessellation of the GM white matter boundary, automated topology correction, and surface deformation following intensity gradients to optimally place the grey/white and grey/ cerebrospinal fluid borders at the location where the greatest shift in intensity defines the transition to the other tissue class. Once the cortical models are complete, a number of deformable procedures can be performed for further data processing and analysis, including surface inflation, registration to a spherical atlas (which utilised individual cortical folding patterns to match cortical geometry across subjects), parcellation of the cerebral cortex into units based on gyral and sulcal structure, and creation of a variety of surface based data including maps of curvature and sulcal depth represented as the local gyrification index (LGI). The LGI is a metric that represents the extent of cortical folding, that is, the larger the folding the higher the LGI (http:// surfer.nmr.mgh.harvard.edu/fswiki/LGI). The LGI is the ratio of the total pial cortical surface over the surface of the brain delineated on two-dimensional coronal sections.

Any misclassification of tissue types was corrected by minimal manual adjustment. The cortical thickness was measured as the distance from the GM/white matter boundary to the corresponding pial surface. The measured cortical thickness values were applied to the white matter surface. By inflating the cortical sulci, an inflated white matter surface model was created and used for statistical analysis. We also segmented and calculated the volume of subcortical structures including the hippocampus, amygdala, thalamus, pallidum, putamen, accumbens, caudate, cerebellum and brain stem.

\section{Statistical analysis}

Analyses of clinical and behavioural data were carried out using SPSS V.19.0. Group differences in demographic, clinical and cognitive characteristics were analysed using multivariate analysis of variance (MANOVA) for normally distributed variables. Post hoc analysis was carried out using Dunnet's T3 method (unequal variances) and Bonferroni correction (equal variances).

For imaging data, a surface-based Gaussian smoothing kernel of full-width half maximum (FWHM) of $20 \mathrm{~mm}$ was used before analysis was carried out. We tried different smoothing levels of $10,15,20,25$ and $30 \mathrm{~mm}$. We found that at $20 \mathrm{~mm}$ FWHM, distinct clusters with unique peaks could be identified. Using a general linear model, we carried out group comparisons controlling for age and gender. We investigated the regional difference in cortical thickness and surface between groups. Correlations between thickness and selected behavioural and clinical measures were also modelled. The offset and slope are subject-independent regression coefficients estimated separately for each vertex using a general linear model while controlling for age and gender. Correlation coefficients were calculated from the slope and mapped on the surface. The level of significance was set at $\mathrm{p}<0.05$ corrected for multiple comparison using the false discovery rate. ${ }^{25}$

\section{Modelling disease progression}

Advancing disease stage from EPD to PDD is a reflection of disease progression. In order to model disease progression we compared PD subjects at difference disease stages. A similar approach was used by Braak to demonstrate the pathological progression of PD. ${ }^{4}{ }^{26} \mathrm{H} \& \mathrm{Y}$ staging is simple and widely used but is inherently non-linear ${ }^{27}$ and does not take into account cognitive impairment, one of the targets of this study. For this reason we characterised disease stage based on H\&Y stage, cognitive impairment and disease duration. This method was based on plotting the raw data of all these variables and ensuring that no subject belonged to more than one group. Based on this, as explained in the Methods section, we divided the PD sample into three groups: group $1(n=24)$ consisting of EPD subjects with $H \& Y$ stage $\leq 2$, disease duration $\leq 5$ years, absence of motor fluctuations and absence of dementia (MMSE $\geq 26$ ); group $2(n=18)$ consisting of patients with moderate PD without dementia (MPD) with H\&Y stage 2-4, disease duration $>5$ years and absence of dementia (MMSE $\geq 26$ ); and group 3 $(n=15)$ consisting of PD patients with dementia (PDD) with $\mathrm{H} \& \mathrm{Y}$ stage $>3$, disease duration $>5$ years and presence of dementia (according to DSM-IV-TR and MDS criteria and scoring $<26$ on the MMSE).

Under this model, MPD and PDD subjects were older than those with EPD. Therefore, we also divided the control group into younger healthy control (YHC) and older healthy control (OHC) subgroups, to match the EPD and MPD/PDD groups, respectively. This allowed us to cancel out the effect of age on group comparisons. In addition, we considered age and gender as covariates of no interest in all group comparisons.

\section{RESULTS}

The clinical, cognitive and demographic characteristics of the study cohort are shown in table 1 . There were no significant differences in gender proportion, years of education, disease duration or age between patient groups and their respective control groups, or when the MPD and PDD groups were compared. Overall cognitive performance, assessed by the MMSE, showed no difference between the YHC and EPD, or the OHC and MPD groups. As expected, the PDD group showed significant cognitive decline compared to the other groups $(\mathrm{p}<0.001)$.

\section{Cortical thickness across groups}

Group comparison of mean cortical thickness generally showed no significant differences between YHC, EPD and OHC. However, cortical thickness was significantly $(\mathrm{p}<0.01)$ reduced in MPD compared to OHC and in PDD compared to MPD (upper half of table 2).

Vertex-wise comparison of cortical thickness between EPD and YHC showed no difference in any cortical area.

Table 2 Global mean cortical thickness and surface in Parkinson's disease

\begin{tabular}{llll}
\hline & Mean \pm SEM & Minimum & Maximum \\
\hline Thickness $(\mathrm{mm})$ & & & \\
YHC & $2.52 \pm 0.03$ & 2.23 & 2.84 \\
EPD & $2.54 \pm 0.04$ & 2.16 & 2.96 \\
OHC & $2.52 \pm 0.04$ & 2.25 & 2.81 \\
MPD & $2.44 \pm 0.04^{*}$ & 2.14 & 2.77 \\
PDD & $2.34 \pm 0.05^{*}$ & 1.99 & 2.69 \\
Surface $\left(\mathrm{mm}^{2}\right)$ & & & \\
YHC & $4957 \pm 196$ & 6655 & 8877 \\
EPD & $4893 \pm 199$ & 6604 & 8719 \\
OHC & $4762 \pm 218$ & 6075 & 7779 \\
MPD & $4544 \pm 255$ & 6174 & 8637 \\
PDD & $4242 \pm 205$ & 5650 & 6972 \\
\hline
\end{tabular}

Mean cortical thickness in MPD was significantly $(p<0.01)$ less than in $\mathrm{OHC}$, and more than in PDD $(p<0.01)$. No significant changes in the mean cortical surface were seen.

EPD, early Parkinson's disease; MPD, moderate Parkinson's disease; OHC, old healthy control; PDD, Parkinson's disease with dementia; YHC, young healthy control. 
Comparison between MPD and OHC showed specific regional thinning in both hemispheres, which was more prominent in the right than in the left (figure 1A). In the right hemisphere, changes were located in the supplementary motor cortex, posterior cingulate, superior frontal, premotor, dorsolateral prefrontal, supramarginal, angular and inferior temporal cortex. The left hemisphere also showed significant cortical thinning in the precuneus, premotor cortex, superior frontal gyrus, dorsolateral prefrontal cortex and fusiform cortex.

Comparison between PDD and OHC showed extensive thinning in the frontal, temporal and posterior parietal cortices (figure 1B). More specifically, the right hemisphere showed a severe reduction in cortical thickness in the anterior pole, dorsolateral prefrontal, premotor, superior frontal, mid and posterior cingulated, precuneus, supramarginal, angular, fusiform and entire temporal cortex. An almost similar pattern was seen in the left hemisphere but with a lesser extent.

Comparison between MPD and PDD showed regional changes in the right hemisphere including the posterior cingulate, premotor, lateral temporal, inferior temporal and anterior temporal pole cortex. In the left hemisphere, changes were limited to the supplementary motor, superior frontal and temporal pole cortex (figure $1 \mathrm{C}$ ). The exact anatomical location, cluster size, max $\mathrm{p}$ value and coordinates of the results can be found in the online supplementary table.

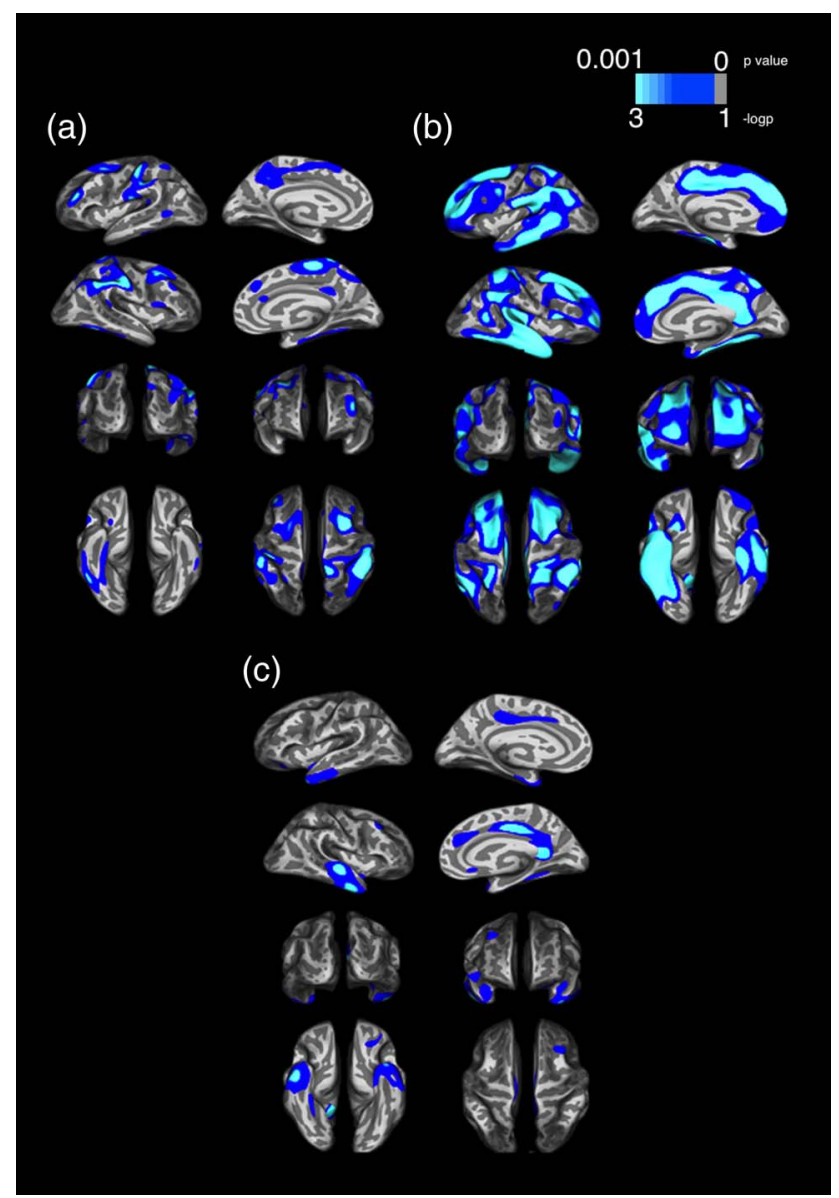

Figure 1 Vertex-wise comparisons between (A) control and moderate Parkinson's disease (MPD), (B) control and Parkinson's disease with dementia (PDD) and (C) MPD and PDD. There is a pattern of extension of the cortical area affected from early Parkinson's disease (almost none) to PDD (prefrontal, temporal and occipito-parietal regions). The colour scale bar shows the logarithmic scale of $p$ values $\left(-\log ^{10}\right)$.
Cortical surface area across groups

Group comparison of cortical surface areas showed a trend towards a reduction in PD groups compared to controls, even though this did not reach the level of significance (see lower half of table 2). To ensure that global brain volume did not affect our result, we took brain volume as a confounder in the group comparisons but the result did not differ.

\section{Local cortical gyrification index}

Group comparison of the LGI showed no differences between groups.

\section{Modelling disease stage}

To assess the relationship between cortical thickness and disease stage, we used a linear regression model with cortical thickness as independent variable and disease stage as dependent variable adjusting for age and gender. This analysis included all subjects. We found that cortical thickness in the occipito-parietal, fusiform, premotor, precuneus, temporal and prefrontal cortices was associated with disease stage (figure 2A). This correlation appears to be more extensive and stronger in the right hemisphere than in the left. In addition, disease stages correlated with LGI in the left rostral middle frontal, left superior parietal, left caudal middle frontal, left superior frontal, left supramarginal, left lateral occipital, left inferior parietal, right superior frontal and right superior parietal areas (see online supplementary figure e-1). The exact anatomical location, cluster size, max $\mathrm{p}$ value and coordinates of the results can be found in the online supplementary table.

\section{Motor and cognitive correlates of cortical thinning}

Looking at specific cognitive and motor correlates of cortical thickness measures, we found that UPDRS-III score did not show any significant correlation with cortical thickness. However, MMSE score showed a strong positive correlation in supramarginal, dorsolateral prefrontal, orbitofrontal and temporal cortices (figure 2B). This correlation was stronger in the left hemisphere than in the right. The exact anatomical location, cluster size, max $\mathrm{p}$ value and coordinates of the results can be found in the online supplementary table.

\section{Volumetry of subcortical and other deep structures}

Group comparison of the volume of the basal ganglia, hippocampus and amygdala between EPD and controls showed no significant difference. EPD and MPD also did not show any significant difference in the volume of these structures. However, PDD subjects had a smaller amygdala and hippocampus compared to MPD, EPD and controls $(\mathrm{p}<0.05$ using Bonferroni correction).

The correlation analyses in PD patients showed that there was a significant negative correlation between left caudate volume and UPDRS-III $(\mathrm{p}<0.03, \mathrm{r}=0.6)$ and between right putamen volume and H\&Y stages $(p<0.002, r=0.73)$; that is, the lower the left caudate and right putamen volume in PD patients, the higher the UPDRS-III and the H\&Y stages, respectively.

\section{Classifying patients with dementia}

Based on the regions that showed significant differences between MPD and PDD, we performed receiver operating characteristics (ROC) and discriminant analysis with a leave-one-out cross validation test. The ROC curve for the mean cortical thickness of areas as well as the volume of subcortical grey structures which showed significant difference between subjects with 
Figure 2 Vertex-wise correlations between cortical thickness and disease modelling stages (A) and Mini-Mental State Examination scores (B). There is bilateral but asymmetrical cortical thinning associated with advancement of Parkinson's disease stage. The colour scale bar shows the logarithmic scale of $p$ values $\left(-\log ^{10}\right)$. Red indicates positive and blue negative correlation.

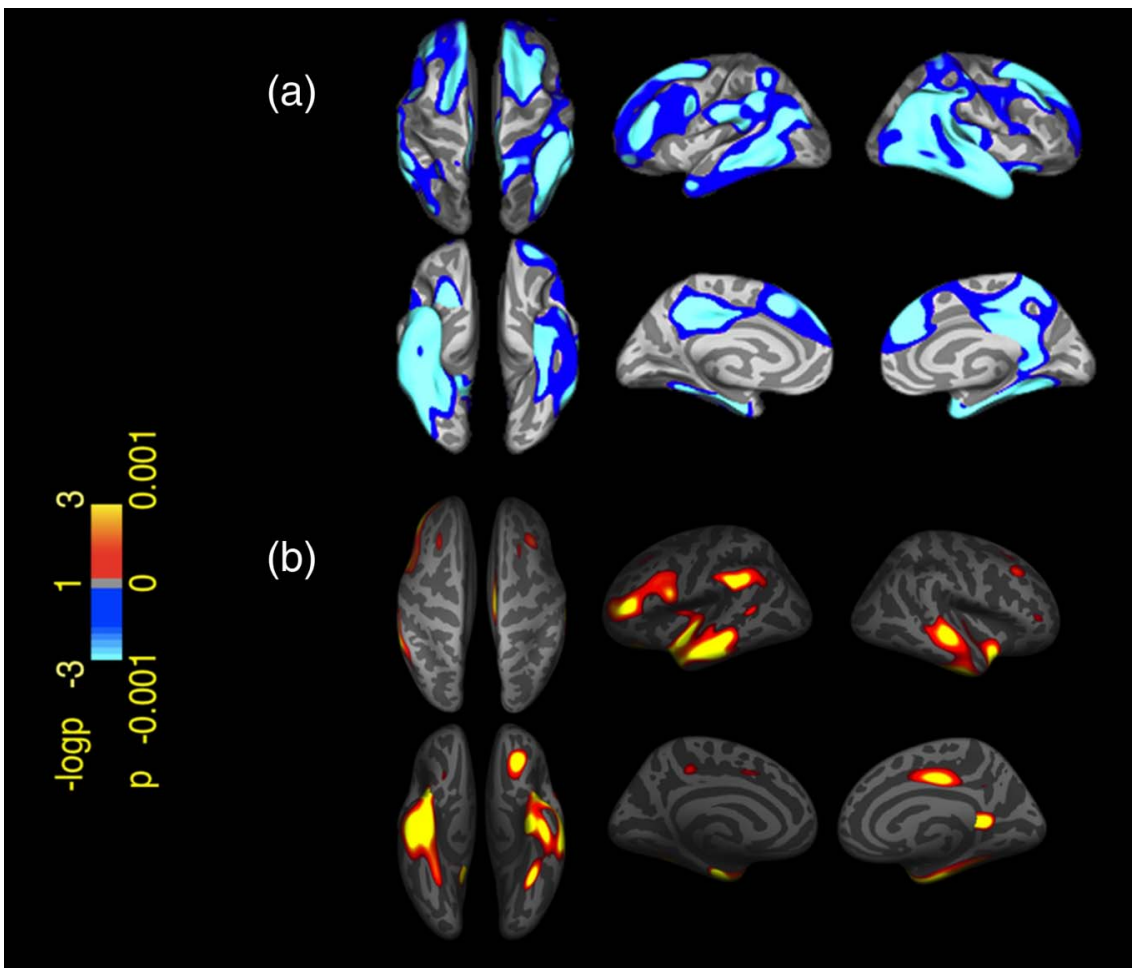

and without dementia, are shown in the upper graph of online supplementary figure e- 2 . We found that the volume of the cerebral cortex, hippocampi and amygdala, as well as the mean thickness of the right superior frontal cortex, right posterior cingulate, right inferior temporal, right parahippocampus, right isthmus cingulate, left temporal pole and left entorhinal cortex are also useful markers to differentiate PD subjects with dementia from PD subjects without dementia (lower graph of online supplementary figure e-2). Discriminant analysis (table 3) showed that the above measures correctly identify at least $80 \%$ of subjects with dementia. In fact, measuring the thickness of the cerebral cortex and the volume of the hippocampi alone provides similar accuracy for the identification of patients with dementia. The cut-offs to discriminate subjects with dementia from those without dementia are $2.4 \mathrm{~mm}$ for mean cortical thickness and $3200 \mathrm{~mm}^{3}$ for hippocampal volume.

\section{DISCUSSION}

This study shows that the cerebral cortex is regionally affected across PD stages which may reflect disease progression. Patients with EPD have no significant cortical thinning, while patients with moderate and severe PD demonstrate remarkable

Table 3 Discriminant analysis of mean cortical thickness

\begin{tabular}{lllll}
\hline & EPD & MPD & PDD & Total \\
\hline EPD & $18(75 \%)$ & $5(20.8 \%)$ & $1(4.2 \%)$ & $24(100 \%)$ \\
MPD & $6(33.3 \%)$ & $7(38.9 \%)$ & $5(27.8 \%)$ & $18(100 \%)$ \\
PDD & 0 & $3(20 \%)$ & $12(80 \%)$ & $15(100 \%)$
\end{tabular}

Discriminant analysis with a leave-one-out cross validation test for the mean volume of the left hippocampus, and the cortical thickness of the left temporal pole, left entorhinal cortex, right hippocampus, right superior frontal, right posterior cingulate, right inferior temporal, right parahippocampal and right isthmus cingulate. Values in parentheses refer to the percentage of subjects correctly identified using the above measures.

EPD, early Parkinson's disease; MPD, moderate Parkinson's disease; PDD, Parkinson's disease with dementia. cortical involvement, with this pattern of ascending cortical involvement being consistent with Braak's hypothesis of PD pathological progression. This is further supported by significant associations between cortical thickness and both disease stages and global cognitive performance, showing that as the disease advances, cortical degeneration extends further, and that the sequence of this extension is generally consistent with that described by Braak.

Cortical thinning in PD correlated with disease stage and initially involved the bilateral supramarginal (area 40), bilateral premotor, bilateral dorsolateral prefrontal, left precuneus cortex and right inferior-medial temporal cortex. As the disease stage increased, cortical regions adjacent to these areas as well as homologous contralateral regions became involved. In the initial stages, there was a right hemispheric predilection, but as the disease progressed the cortical involvement became bilateral. There are inconsistent results in terms of structural hemispheric asymmetry in PD. Some authors found greater right and some others left hemisphere involvement. ${ }^{8}$ A recent pilot study also reported that PD patients without dementia showed significant cortical thinning in the right middle temporal gyrus. ${ }^{28}$ The underlying cause for such hemispheric predilection is unclear. Thus far there is no hard evidence to show that this predilection tomatology. There are too few studies for a strong conclusion about a possible right hemispheric predilection in PD.

Presence of dementia was associated with extensive thinning of the dorsal and medial frontal cortex as well as the temporal cortex and posterior parietal cortex. Clearly, the development of dementia in PD is a gradual process. However, the comparison between aged-matched PD subjects with and without dementia showed that dementia was associated mainly with the extent of cortical thinning in the temporal cortex as well as the posterior cingulate gyrus. This indicates that involvement of the temporal cortex is essential in the development of dementia in PDD. This is further supported by a strong negative correlation between cortical thickness in the temporal cortex and is related to handedness or the predominant side of PD symp- 
MMSE score as well as with disease stage. A community-based longitudinal study of newly diagnosed PD patients showed that pentagon copying and semantic verbal fluency were the most significant neuropsychological predictors of cognitive decline leading to dementia. ${ }^{29}$ Our study, thus, provides quantitative evidence to support earlier works suggesting that defects in cortical functions are responsible for the development of dementia. $^{29} 30$

Regarding motor severity as measured by the UPDRS-III subscale, there was no correlation with cortical thickness, but the associations with subcortical (basal ganglia) structures were robust. Indeed, in the PD patients there was a significant negative correlation between left caudate volume and UPDRS-III scores. This is in keeping with the view that motor disability in $\mathrm{PD}$ is mainly associated with impairment of subcortical regions, namely nigrostriatal and nigrothalamic connections.

The pattern of cortical thinning in our cohort was consistent with Braak's hypothesis of PD pathological progression, according to which the pathology has reached the basolateral nuclear complex of the amygdala, the striatum and thalamic midline as well as intralaminar nuclei by the time PD is clinically diagnosed. ${ }^{31}$ As the disease progresses, the pathology encroaches upon the cerebral cortex, mainly the anteromedial temporal mesocortex, directly connected with the prefrontal cortex, posterior cingulate and high order sensory association areas. ${ }^{3}$ In fact, involvement of the temporal mesocortex is followed by spread of the lesions to the high order association fields of the neocortex. ${ }^{32}$ However, although our results agree with this model, it is only possible to conclude that they support progressive cortical involvement in PD and related dementia, since pathologies other than Lewy pathology, such as Alzheimer's disease, have also been related to PDD and might well account for the cortical thinning we have observed. ${ }^{6} 733$

We showed that mean cortical thickness and hippocampal volume can reliably classify $80 \%$ of subjects with dementia (sensitivity), with specificity of around $85 \%$ in our sample. Recent studies support involvement of the hippocampus as a biomarker of cognitive decline and progression to dementia in $\mathrm{PD},{ }^{13}{ }^{14}$ but our study is the first to show the value of cortical thickness for classifying PD patients with dementia. This measure may be even more useful as some of the moderate PD subjects had some degree of cognitive impairment that the MMSE failed to identify. The MMSE, although used commonly, has around $70 \%$ sensitivity for identifying dementia in PD. ${ }^{34}$ On a practical level, thus, normal cortical thickness in EPD may provide a potential window of opportunity for the use of diseasemodifying drugs for the prevention of dementia in PD. Moreover, cortical thinning may be a useful measure for correctly selecting patients for deep brain stimulation, as those with cognitive impairment are usually excluded from such treatment.

Group comparisons of cortical surface did not show significant changes, but the trend was in line with our observations of cortical thickness analysis. One possible explanation for this could be that cortical thickness represents an early stage of cortical degenerative processes which may not necessarily affect the surface area. ${ }^{35}$ It is possible that changes in cortical surface reflect global brain volume changes. However, in our study this was not the case as we re-analysed the data taking brain volume as a covariate of no interest, but the result remained the same. The disease stages correlated with the gyrification index in several cortical areas. This finding is in line with previous studies suggesting that the neurodegenerative process in PD is reflected by cortical folding and especially cortical thickness measurements. ${ }^{12}$ However, it should be taken into account that, although such measurements are presumed to detect changes in the cortical GM layer of PD patients, we would need pathological postmortem confirmation of these changes. Therefore, future studies assessing a wide sample of PD patients in different stages of the disease and involving postmortem pathological confirmation, are essential to further assess the relationship between the clinical course and progression to dementia.

One potential confounder in our model of disease progression is the effect of normal aging on cortical thinning. To minimise this we took age and gender as covariates of no interest in all our analysis. In addition, the pattern of cortical thinning that we found is different from that previously reported for normal aging. ${ }^{36}$ While we provided a practical model for disease staging reflecting disease progression by comparing PD at different stages, this crosssectional design does not replace a well-designed longitudinal study which is essential to confirm our findings.

\section{Author affiliations}

${ }^{1}$ Department of Neurology, University of Nottingham, Nottingham, UK ${ }^{2}$ Institute for Cognitive Science Studies, Tehran, Iran

${ }^{3}$ Department of Psychiatry and Clinical Psychobiology, University of Barcelona, Barcelona, Catalonia, Spain

${ }^{4}$ Centro de Investigación en Red de Enfermedades Neurodegenerativas (CIBERNED), Barcelona, Catalonia, Spain

${ }^{5}$ Department of Methods and Experimental Psychology, Faculty of Psychology and Education, University of Deusto, Bilbao, Basque Country, Spain

${ }^{6}$ Parkinson's Disease and Movement Disorders Unit, Neurology Service, Institut Clínic de Neurociències (ICN), Institute of Biomedical Research August Pi i Sunyer (IDIBAPS), Hospital Clínic de Barcelona, Barcelona, Catalonia, Spain

${ }^{7}$ Centre for Functional MRI of the Brain, University of Oxford, Oxford, UK

${ }^{8}$ Centre de Diagnòstic per la Imatge Hospital Clínic de Barcelona (CDIC), Hospital

Clínic de Barcelona, IDIBAPS, Barcelona, Catalonia, Spain

Contributors Research project: MZ, CJ, MJM, conception; NI-B, YC, CJ, ET, MJM, organisation; NI-B, YC, CJ, NB, ET, MJM, execution. Statistical analysis: MZ, NI-B, $\mathrm{MH}$, design and execution; MZ, YC, CJ, NB, ET, MJM, review and critique. Manuscript: MZ, NI-B, writing of the first draft; $Y C, C J, N B, E T, M J M$, review and critique.

Funding NI-B received funding from the Spanish Ministry of Education and Science (AP 2005-019) and the Center for Biomedical Investigation of Neurodegenerative Diseases (CIBERNED); YC and MJM received funding from Fundació la Marató de TV3 2006 (N-2006-TV060510); CJ received funding from the Spanish Ministry of Education and Science (SAF05-007340; SAF07-66077, PSI2010-16174) and the Government of Catalonia (2005SGR00855, 2009SGR0941, UNI/2001-2004, Red CIEN IDIBAPS-ISCIII RTIC C03/06); NB received funding from the Spanish Ministry of Education and Science and the Spanish Ministry of Health; and ET received funding from CIBERNED and the Government of Catalonia (2005SGR0836, 2009SGR0836, Red CIEN IDIBAPS-ISCIII RTIC C03/06).

Competing interests None.

Ethics approval The University of Barcelona approved this study.

Provenance and peer review Not commissioned; externally peer reviewed.

Open Access This is an Open Access article distributed in accordance with the Creative Commons Attribution Non Commercial (CC BY-NC 3.0) license, which permits others to distribute, remix, adapt, build upon this work non-commercially, and license their derivative works on different terms, provided the original work is properly cited and the use is non-commercial. See: http://creativecommons.org/ licenses/by-nc/3.0/

\section{REFERENCES}

1 Aarsland D, Andersen K, Larsen JP, et al. Prevalence and characteristics of dementia in Parkinson disease: an 8-year prospective study. Arch Neurol 2003;60:387-92

2 Hely MA, Reid WG, Adena MA, et al. The Sydney multicenter study of Parkinson's disease: the inevitability of dementia at 20 years. Mov Disord 2008;23:837-44

3 Braak H, Del Tredici K, Rüb U, et al. Staging of brain pathology related to sporadic Parkinson's disease. Neurobiol Aging 2003;24:197-211.

4 Braak H, Rub U, Steur EN Jansen, et al. Cognitive status correlates with neuropathologic stage in Parkinson disease. Neurology 2005;64:1404-10.

5 Ballard C, Ziabreva I, Perry R, et al. Differences in neuropathologic characteristics across the Lewy body dementia spectrum. Neurology 2006;67:1931-4. 
6 Halliday G, Hely M, Reid W, et al. The progression of pathology in longitudinally followed patients with Parkinson's disease. Acta Neuropathol 2008;115:409-15.

7 Compta Y, Parkkinen L, O'Sullivan SS, et al. Lewy- and Alzheimer-type pathologies in Parkinson's disease dementia: which is more important? Brain 2011;134(Pt 5): 1493-505.

8 Ibarretxe-Bilbao N, Tolosa E, Junque C, et al. MRI and cognitive impairment in Parkinson's disease. Mov Disord 2009;24(Suppl 2):S748-53.

9 Ibarretxe-Bilbao N, Junque C, Marti MJ, et al. Brain structural MRI correlates of cognitive dysfunctions in Parkinson's disease. J Neurol Sci 2011;310:70-4.

10 Lyoo $\mathrm{CH}$, Ryu YH, Lee MS. Topographical distribution of cerebral cortical thinning in patients with mild Parkinson's disease without dementia. Mov Disord 2010;25:496-9.

11 Jubault T, Gagnon JF, Karama S, et al. Patterns of cortical thickness and surface area in early Parkinson's disease. Neuroimage 2011;55:462-7.

12 Pereira JB, Ibarretxe-Bilbao N, Marti MJ, et al. Assessment of cortical degeneration in patients with Parkinson's disease by voxel-based morphometry, cortical folding, and cortical thickness. Hum Brain Mapp 2012:33:2521-34.

13 Weintraub D, Dietz N, Duda JE, et al. Alzheimer's disease pattern of brain atrophy predicts cognitive decline in Parkinson's disease. Brain 2012;135(Pt 1):170-80.

14 Weintraub D, Doshi J, Koka D, et al. Neurodegeneration across stages of cognitive decline in Parkinson disease. Arch Neurol 2011;68:1562-8.

15 Dickerson BC, Feczko E, Augustinack JC, et al. Differential effects of aging and Alzheimer's disease on medial temporal lobe cortical thickness and surface area. Neurobiol Aging 2009;30:432-40.

16 Rosas HD, Salat DH, Lee SY, et al. Cerebral cortex and the clinical expression of Huntington's disease: complexity and heterogeneity. Brain 2008;131(Pt 4):1057-68.

17 Salat DH, Lee SY, van der Kouwe AJ, et al. Age-associated alterations in cortical gray and white matter signal intensity and gray to white matter contrast. Neuroimage 2009;48:21-8.

18 Ibarretxe-Bilbao N, Junque C, Tolosa E, et al. Neuroanatomical correlates of impaired decision-making and facial emotion recognition in early Parkinson's disease. Eur J Neurosci 2009;30:1162-71

19 Compta Y, Marti MJ, Ibarretxe-Bilbao N, et al. Cerebrospinal tau, phospho-tau, and beta-amyloid and neuropsychological functions in Parkinson's disease. Mov Disord 2009:24:2203-10.

20 Compta Y, Ibarretxe-Bilbao N, Pereira JB, et al. Grey matter volume correlates of cerebrospinal markers of Alzheimer-pathology in Parkinson's disease and related dementia. Parkinsonism Relat Disord 2012;18:941-7.

21 Daniel SE, Lees AJ. Parkinson's Disease Society Brain Bank, London: overview and research. J Neural Transm Supp/ 1993;39:165-72.
22 Litvan I, Bhatia KP, Burn DJ, et al. Movement Disorders Society Scientific Issues Committee report: SIC Task Force appraisal of clinical diagnostic criteria for Parkinsonian disorders. Mov Disord 2003:18:467-86.

23 Fischl B, Dale AM. Measuring the thickness of the human cerebral cortex from magnetic resonance images. Proc Natl Acad Sci USA 2000;97:11050-55.

24 Fischl B, Sereno MI, Tootell RB, et al. High-resolution intersubject averaging and a coordinate system for the cortical surface. Hum Brain Mapp 1999;8: 272-84.

25 Genovese CR, Lazar NA, Nichols T. Thresholding of statistical maps in functional neuroimaging using the false discovery rate. Neuroimage 2002;15:870-8.

26 Muller CM, de Vos RA, Maurage CA, et al. Staging of sporadic Parkinson disease-related alpha-synuclein pathology: inter- and intra-rater reliability. J Neuropathol Exp Neurol 2005;64:623-8.

27 Goetz CG, Poewe W, Rascol 0, et al. Movement Disorder Society Task Force report on the Hoehn and Yahr staging scale: status and recommendations. Mov Disord 2004;19:1020-8.

28 Pellicano C, Assogna F, Piras F, et al. Regional cortical thickness and cognitive functions in non-demented Parkinson's disease patients: a pilot study. Eur J Neurol 2012;19:172-5.

29 Williams-Gray $\mathrm{CH}$, Foltynie T, Brayne $\mathrm{CE}$, et al. Evolution of cognitive dysfunction in an incident Parkinson's disease cohort. Brain 2007:130(Pt 7):1787-98.

30 Pagonabarraga J, Kulisevsky J, Llebaria G, et al. Parkinson's disease-cognitive rating scale: a new cognitive scale specific for Parkinson's disease. Mov Disord 2008;23:998-1005.

31 Del Tredici K, Rub U, De Vos RA, et al. Where does parkinson disease pathology begin in the brain? I Neuropathol Exp Neurol 2002;61:413-26.

32 Braak H, Muller CM, Rub U, et al. Pathology associated with sporadic Parkinson's disease — where does it end? J Neural Transm Supp/ 2006;70:89-97.

33 Sabbagh MN, Adler CH, Lahti TJ, et al. Parkinson disease with dementia: comparing patients with and without Alzheimer pathology. Alzheimer Dis Assoc Disord 2009;23:295-7.

34 Hoops $\mathrm{S}$, Nazem S, Siderowf AD, et al. Validity of the MoCA and MMSE in the detection of $\mathrm{MCl}$ and dementia in Parkinson disease. Neurology 2009;73: $1738-45$.

35 Nopoulos PC, Aylward EH, Ross CA, et al. Cerebral cortex structure in prodromal Huntington disease. Neurobiol Dis 2010:40:544-54.

36 Salat DH, Buckner RL, Snyder AZ, et al. Thinning of the cerebral cortex in aging. Cereb Cortex 2004;14:721-30. 